NASA Technical Memorandum 4030

\title{
Non-Newtonian Gravity or Gravity Anomalies?
}

David P. Rubincam, B. Fong Chao, Kenneth H. Schatten, and William W. Sager

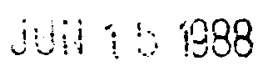

LANGLEY RESEARCH CENTER

LIBRARY, NASA

HAMPTON, VIREINIA' 
NASA Technical Memorandum 4030

\section{Non-Newtonian Gravity or Gravity Anomalies?}

David P. Rubincam, B. Fong Chao, and Kenneth H. Schatten

Goddard Space Flight Center

Greenbelt, Maryland

William W. Sager

Texas AEM University

College Station, Texas

\section{N/SA}

National Aeronautics

and Space Administration

Scientific and Technical Information Division 
NEWTONIAN GRAVITY OR GRAVITY ANOMALIES?

Stacey and coworkers (Stacey and Tuck, 1981; Stacey et al., 1981; Stacey, 1983; Holding and Tuck, 1984; Holding et al., 1986), and Hsui (1987) all present tentative evidence that gravity is non-Newtonian. Their geophysically determined $G$, the universal constant of gravitation, is on the order of 1 per cent larger than the laboratory value of $6.672 \pm 0.004 \times 10^{-11} \mathrm{~m}^{3}$ $\mathrm{kg}^{-1} \mathrm{~s}^{-2}$. Their values are derived from measurements of the gravitational acceleration $\mathrm{g}$ conducted in mine shafts, boreholes, and ocean depths, and a (Newtonian) theory of how $g$ varies inside the Earth.

Briefly, the technique is to assume a rotationally flattened Earth and to remove ellipsoidal shells above the depth at which $\mathrm{g}$ is measured. Since the gravitational attraction is zero inside a homogeneous ellipsoidal shell, only the ellipsoid below the gravimeter contributes to $\mathrm{g}$. By correcting for the mass of the shells, one knows how much mass remains in the ellipsoid, and therefore its pull on the gravimeter. Departures from the expected Newtonian values at varying depths inside the Earth provide the evidence for non-Newtonian gravity.

However, it is the local density that appears in the equations of the theory, and densities in the local environment are carefully measured. But the local density is not usually characteristic of the average density of the shell which must be used in computing its mass. For example, the local density of a borehole on a continent is about $2800 \mathrm{~kg} \mathrm{~m}^{-3}$, while at a depth just below sea level the average density will be approximately (0.7) $1000+$ $(0.3) 2800 \simeq 1540 \mathrm{~kg} \mathrm{~m}^{-3}$, reflecting the various proportions of water and continental rock. This example illustrates that the average density can be much different from the local density.

We wish to comment on the resolution of this apparent contradiction. More importantly, we will demonstrate how large-scale density anomalies in the Earth (scale length $>500 \mathrm{~km}$ ), if not corrected for, may masquerade as non-Newtonian gravity, and provide spurious evidence for a "fifth force" . We do this by showing that simplified Earth model containing only long-wavelength gravity anomalies can affect the measurement of $G$ at the level reported for non-Newtonian behavior.

In illustrating our remarks we assume the following: the Earth is nonrotating and spherical in shape with radius $R$ and mass $M$. For simplicity in our expressions we shall not consider the centrifugal acceleration and flattening due to rotation because they can be analytically corrected for (Stacey et al., 1981; Dahlen, 1982). We further assume an Earth model whose density distribution $\rho(\underline{r})$ has zero multipoles beyond a certain degree $I$ but is otherwise identical to the Earth, so that all the sums appearing later are finite. In terms of spherical hamonics, $\rho(\underline{r})$ can thus be written as 


$$
\rho(\underline{r})=\sum_{l=0}^{L} \sum_{m=0}^{l} \sum_{i=1}^{2} \rho_{1 m i}(r) \mathrm{Y}_{1 m i}(\Omega)
$$

where

$$
\begin{aligned}
& \mathrm{Y}_{\operatorname{lm} 1}(\Omega)=\mathrm{P}_{1 \mathrm{~m}}(\cos \theta) \operatorname{cosm} \lambda \\
& \mathrm{Y}_{1 \mathrm{~m} 2}(\Omega)=\mathrm{P}_{1 \mathrm{~m}}(\cos \theta) \sin \lambda
\end{aligned}
$$

and $P_{1 m}$ is the nomalized associated Legendre function of degree 1 and order $m$, and $\underline{r}=(r, \Omega)$ where $\Omega$ is an abbreviation for both the co-latitude $\theta$ and the longitude $\lambda$. The spherical harmonics are orthogonal and we adopt the nomalization commonly used in geophysics:

$$
\int \mathrm{Y}_{1 \mathrm{mi}}(\Omega) \mathrm{Y}_{\mathrm{I}^{\prime} \mathrm{m}^{\prime} \mathrm{i}^{\prime}}(\Omega) \mathrm{d} \Omega=4 \pi \delta_{1 l^{\prime}}, \delta_{\mathrm{mm}^{\prime}} \delta_{\mathrm{ii}^{\prime}}
$$

over the unit sphere. Apart from the rotational flattening, only the $1=0$ (monopole) terms have been considered previously. We shall consider the $0<1 \leq L$ terms as additional density anomalies. In the following we fix the origin at the Earth's center of mass so that $l=1$ terms vanish. For $1=2,4, m=0$ terms we consider only the departure of the Earth from the state of a hydrostatic flattening due to rotation (e.g., Jeffreys, 1976). Also, ignoring any polar motion (of the rotational axis) which only produces a centrifugal acceleration on the order of $10^{-7} \mathrm{~m} \mathrm{~s}^{-2}$ (or $10 \mu \mathrm{gal}$ ), we set $1=2, m=1$ terms to zero.

The gravitational potential at a point inside the Earth $(r<R)$, satisfying Poisson's equation $\nabla^{2} U(\underline{r})=-4 \pi G \rho(\underline{r})$, is

$U(\underline{I})=4 \pi G \sum_{\operatorname{lmi}} \frac{1}{2 l+1} Y_{1 m i}(\Omega)\left[r^{-1-1} \int_{0}^{r} s^{l+2} \rho_{1 m i}(s) d s+r^{l} \int_{r}^{R} s^{-1+1} \rho_{I m i}(s) d s\right]$

Writing the first integral in equation (4) as $\int_{0}^{R}-\int_{r}^{R}$, and considering the radial component by differentiation leads to 


$$
\begin{gathered}
g(\underline{I}) \simeq-\frac{\partial U(\underline{r})}{\partial r}=\frac{G M}{R^{2}} \sum_{l m i}(1+1) Y_{l m i}(\Omega)\left(\frac{R}{r}\right)^{l+2} C_{l m i} \\
-4 \pi G \underset{l m i}{\sum} \frac{1}{2 l+1} Y_{l m i}(\Omega) \int_{r}^{R} \rho_{l m i}(s)\left[(1+1)\left(\frac{s}{r}\right)^{1+2}+1\left(\frac{s}{r}\right)^{-1+1}\right] d s
\end{gathered}
$$

where the slight difference in the local vertical and radial directions is ignored. The $\mathrm{C}_{l m i}$ are the nomalized multipoles of the density $\rho(\underline{r})$, given by

$$
C_{l m i}=\frac{1}{(2 l+1) M R^{l}} \int_{V} \rho(\underline{r}) r^{l} Y_{1 m i}(\Omega) d V
$$

where $\mathrm{V}$ is the volume of the (spherical) Earth. They arise as coefficients, often called the Stokes coefficients, of the external ( $r>R$ ) gravitational field:

$$
U_{\text {ext }}(\underline{r})=\frac{G M}{R} \sum_{l m i} C_{l m i}\left(\frac{R}{r}\right)^{l+1} Y_{l m i}(\Omega)
$$

and have been routinely determined from geodetic measurements (e.g., Lerch et al., 1981; Rapp and Cruz, 1986).

We now differentiate equation (5) to compare with previous studies which measured the radial gradient of $g(\underline{r})$ :

$$
-\frac{\partial g(\underline{\underline{I}})}{\partial r}=\frac{g_{0}}{R} \tilde{Q}(\underline{r})-4 \pi G[\rho(\underline{r})+\tilde{P}(\underline{r})]
$$

where

$$
\tilde{Q}(\underline{r}) \equiv \sum_{l m i}(l+1)(1+2)\left(\frac{R}{r}\right)^{1+3} \mathrm{Y}_{1 m i}(\Omega) c_{1 m i}
$$




$$
\tilde{\mathrm{P}}(\underline{r}) \equiv \sum_{\operatorname{lmi}} \frac{1}{(2 l+1) r} \int_{\mathrm{r}}^{\mathrm{R}} \rho_{l m i}(s)\left[(1+1)(1+2)\left(\frac{s}{r}\right)^{1+2}-1(1-1)\left(\frac{s}{r}\right)^{-1+1}\right] d s
$$

and $g_{0} \equiv G M / R^{2}=9.8209 \mathrm{~m} \mathrm{~s}^{-2}$ is an accurately determined Earth parameter. Note that the effect of the density anomalies inside the shell (of radius $r$ ) is absorbed in the $\mathrm{C}_{1 \mathrm{mi}}$ coefficients (in $\widetilde{Q}$ ) so that only the density of the shell has to be explicitly considered (in P). In finding $\Delta g$ between two depths, as do stacey et al. (1981), one integrates equation (8). This is why an integral involving the local density $\rho(\underline{r})$ appears, and not the average shell density.

Let us now examine the influence of long-wavelength terms previously not considered. We write $\tilde{P}(\underline{r})=P_{0}(r)+P(\underline{r})$ and $\tilde{Q}(\underline{r})=Q_{0}(r)+Q(\underline{r})$ where subscript 0 indicates the $1=0$ (monopole) terms, and no subscript indicates the sum of all higher-degree terms $(2 \leq 1 \leq L)$. From equation (8), the geophysical solution of $\mathrm{G}$ can then be written as

$$
G=\frac{1}{4 \pi\left[\rho(\underline{r})+P_{0}(r)+P(\underline{r})\right]}\left(\frac{\partial g(\underline{r})}{\partial r}+\frac{g_{0}}{R}\left[Q_{0}(r)+Q(\underline{r})\right]\right)
$$

Previous studies only considered the rotation, the flattening (embodied in an $l=2, m=0$ term), and the $P_{0}(r)$ and $Q_{0}(r)$ terms in their solution of $G$. our equation (11) constitutes a generalization of their formula to include $P(\underline{r})$ and $Q(\underline{r})$ which arise from global density anomalies, and reduces to it when $P(\underline{r})$ and $Q(\underline{r})$ are ignored.

We now examine the correction terms $P(\underline{r})$ and $Q(\underline{r})$. We will estimate their importance in equation (11) for the long-wavelength anomalies in the Earth, for which we have chosen $L=36$, corresponding to a scale length of $>500 \mathrm{~km}$.

We consider $\tilde{P}(\underline{r})$ first. If we assume the continents to have constant densities and vertical boundaries for the depths considered, then $\rho_{\operatorname{lmi}}(r)=$ constant and can be moved outside the integral in equation (10). If $1 \mathrm{z} \ll$ $R$, where $z=R-r$ is the depth, then this integral becomes approximately $2(2 l+1) z$, so that $\tilde{P}(\underline{r}) \simeq 2(z / R) \rho(\underline{r})$. For typical depths (e.g., in a borehole) of $1 \mathrm{~km}, \tilde{P}(\underline{\underline{r}}) \simeq 0.0003 \rho(\underline{r})$ and can be neglected to this order in equation (11).

Under this approximation, the effect of the gravity anomalies on the solution of $\mathrm{G}$, as a function of the geographical location, is

$$
\Delta G(\underline{r}) \simeq g_{0} Q(\underline{r}) / 4 \pi R \rho(\underline{r}) .
$$


This is the error one makes in the solution of $\mathrm{G}$ if the gravity anomalies are ignored. Note that only the multipoles of the density distribution (the $\mathrm{C}_{\text {lmi }}$ coefficients) are involved -- the complete knowledge of the density itself is not necessary.

We next evaluate $Q(\underline{r})$. For the long-wavelength gravity anomalies we are considering $(1 \leq 36)$, and depths $z<R$, the factor $(R / r)^{1+3}$ in equation (9) can be taken to be 1 with an error of $\sim(1+3)(z / R)$. Thus, for depths of $\sim 1$ $\mathrm{km}$, equation (9) may be evaluated at the Earth's surface with only about a $0.6 \%$ error in $Q(\underline{r})$ (not to be confused with the error in the G solution). For the $C_{I m i}$ coefficients we use those of the GEM-10B gravitational field (Lerch et aI., 1981).

Using the resulting $Q(\underline{r})$ in equation (12) gives $\langle\Delta G\rangle / G \simeq 0.438$, where $\langle\Delta G\rangle$ is the root-mean-square of $\Delta G(\underline{r})$ over the Earth's surface, with $\Delta G(\underline{r})$ being computed at the center of $5^{\circ} \times 5^{\circ}$ squares. The gravity anomalyinduced $\Delta G(\underline{r})$ can be positive or negative depending on where the measurement is made. Extreme values of $\Delta G(r) / G$ reach as high as \pm 2.58 for the $G E M-10 B$ field. These percentages are all in the range of the values reported, and demonstrate that long-wavelength anomalies can significantly affect the geophysical measurement of $\mathrm{G}$.

Stacey et al. (1981) have briefly discussed the free-air gravity gradient at the Australian mine site. They found a discrepancy of $8 \times 10^{-8}$ $\mathrm{sec}^{-2}$ between the measured value and the computed value based on the monopole Earth model. Although as great as $10 \%$ of the gravity gradient measured in the mine shaft, it was absorbed into the uncertainty in their quoted final value for $G$. Here we wish to point out the following. The free-air gradient has the same form as our $g_{0} \mathrm{Q}(r) / R$ (but evaluated outside the Earth), and hence the same physical source. The large discrepancy is thus an indication of the importance of this source, which we have identified to be the density anomalies -- not just local but long-wavelength as well. This cannot be treated as an uncertainty but rather a systematic correction that must be made before a meaningful $G$ value can be deduced. Note also that, at the Australian mine site, the sign of the free-air gradient discrepancy is consistent with one that can cause a positive $\Delta G$ measurement as reported (Stacey et al., 1981).

The modest aim of the present paper has been to indicate that even longwavelength gravity anomalies may seriously affect gravity measurements, leading to an incorrect value of G. However, we make no "long-wavelength corrections" for $G$ for any particular location for the real Earth. This is because of the Kaula's rule-of-thumb (Kaula, 1967) which states that the magnitude of the $\mathrm{C}_{1 \mathrm{mi}}$ coefficients decrease as $10^{-5} 1^{-2}$. This empirical law has been shown by Rapp and Cruz (1986) to roughly hold to at least $L=180$. The sum in equation (9) as a result grows rapidly with 1 far past the limit of the GEM-1OB field $(I=36)$. Except for certain locations where the effects for $1 \leq 36$ and $1>36$ happen to have comparable magnitudes and opposite signs, the inclusion of higher degree terms for the real Earth will, in general, greatly augment the values based on GEM-10B. 


\section{References}

Holding, S.C., and G.J. Tuck, A new mine determination of Newtonian gravitational constant, Nature 307, 714-716, 1984.

Holding, S.C., F.D. Stacey, and G.J. Tuck, Gravity in mines -- An investigation of Newton's law, Phys. Rev. D33, 3487-3494, 1986.

Hsui, A.T., Borehole measurement of the Newtonian gravitational constant, Science 238, 881-883, 1987.

Jeffreys, H., The Earth, 6th ed., Cambridge Univ. Press, New York, 1976.

Kaula, W.M., Geophysical implications of satellite determinations of the Earth's gravitational field, space Sc. Rev. 7, 769-794, 1967.

Lerch, F.J., B.H. Putney, C.A. Wagmer, and S.M. Klosko, Goodard Earth Model for oceanographic applications (GEM $10 \mathrm{~B}$ and 10C), Mar. Geod. 5, 145-187, 1981.

Rapp, R.H., and J.Y. Cruz, Spherical harmonic expansions of the Earth's gravitational potential to degree 360 using 30' mean anomalies, Rep. \$376, The Ohio State Univ., 1986.

Stacey, F.D., Subterranean gravity and other deep hole geophysics, in Science Underground, edited by M.M. Nieto, W.C. Haxton, C.M. Hoffiman, E.W. Kolb, V.D. Sandberg, J.W. Toevs, Amer. Inst. Phys., New York, 285-297, 1983.

Stacey, F.D., and G.J. Tuck, Geophysical evidence for non-Newtonian gravity, Nature 292, 230-232, 1981.

Stacey, F.D., G.J. Tuck, S.C. Holding, A.R. Maher, and D. Morris, Constraint on the planetary scale value of the Newtonian gravitational constant from the gravity profile within a mine, Phys. Rev. D23, 1683-1692, 1981. 


\begin{tabular}{|c|c|c|c|}
\hline$\prod_{\substack{\text { Nalional Aeronautics and } \\
\text { Spice Adminustialion }}}$ & \multicolumn{3}{|c|}{ Report Documentation Page } \\
\hline $\begin{array}{l}\text { 1. Report No. } \\
\text { NASA TM-4030 }\end{array}$ & 2. Government Accession No. & \multicolumn{2}{|c|}{ 3. Recipient's Catalog No. } \\
\hline \multirow{2}{*}{\multicolumn{2}{|c|}{$\begin{array}{l}\text { 4. Title and Subtitle } \\
\text { Non-Newtonian Gravity or Gravity Anomalies? }\end{array}$}} & \multicolumn{2}{|c|}{$\begin{array}{l}\text { 5. Report Date } \\
\text { June } 1988\end{array}$} \\
\hline & & \multicolumn{2}{|c|}{$\begin{array}{l}\text { 6. Performing Organization Code } \\
621\end{array}$} \\
\hline \multicolumn{2}{|l|}{ 7. Author(s) } & \multicolumn{2}{|c|}{$\begin{array}{l}\text { 8. Performing Organization Report No. } \\
\text { 88B0104 }\end{array}$} \\
\hline \multicolumn{2}{|c|}{$\begin{array}{l}\text { David P. Rubincam, B. Fong Chao, Kenneth H. Schatten, } \\
\text { and William W. Sager }\end{array}$} & \multicolumn{2}{|c|}{ 10. Work Unit No. } \\
\hline \multirow{3}{*}{\multicolumn{2}{|c|}{$\begin{array}{l}\text { 9. Performing Organization Name and Address } \\
\text { Goddard Space Flight Center } \\
\text { Greenbelt, Maryland } 20771\end{array}$}} & \multirow{2}{*}{\multicolumn{2}{|c|}{ 11. Contract or Grant No. }} \\
\hline & & & \\
\hline & & \multirow{2}{*}{\multicolumn{2}{|c|}{$\begin{array}{l}\text { 13. Type of Report and Period Covered } \\
\text { Technical Memorandum }\end{array}$}} \\
\hline \multirow{2}{*}{\multicolumn{2}{|c|}{$\begin{array}{l}\text { 12. Sponsoring Agency Name and Address } \\
\text { National Aeronautics and Space Administration } \\
\text { Washington, D.C. 20546-0001 }\end{array}$}} & & \\
\hline & & \multicolumn{2}{|c|}{ 14. Sponsoring Agency Code } \\
\hline \multicolumn{4}{|c|}{$\begin{array}{l}\text { William W. Sager is affiliated with the Department of Oceanography and Geophysics } \\
\text { at Texas A\&M University, College Station, Texas. } \\
\text { Authors Rubincam, Chao and Schatten are affiliated wi th Goddard Space Flight } \\
\text { Center. }\end{array}$} \\
\hline \multicolumn{4}{|c|}{$\begin{array}{l}\text { 16. Abstract } \\
\text { Geophysical measurements of G differ from laboratory values, indicating that } \\
\text { gravity may be non-Newtonian. Here we present a spherical harmonic formulation } \\
\text { for the variation of (Newtonian) gravity inside the Earth. Using the GEM-10B } \\
\text { Earth gravitational field model, we illustrate that long-wavelength gravity } \\
\text { anomalies, if not corrected for, may masquerade as non-Newtonian gravity by } \\
\text { providing significant influences upon experimental observations of ag/ar and } \\
\text { G. We also resolve an apparent contradiction in previous studies: local } \\
\text { densities appear in the equations when average densities of layers seem to } \\
\text { be called for. }\end{array}$} \\
\hline $\begin{array}{l}\text { 17. Key Words (Suggested by Author(s)) } \\
\text { non-Newtonian gravity, } \\
\text { gravity anomalies }\end{array}$ & $\begin{array}{l}\text { 18. Dist } \\
\text { Unc }\end{array}$ & d - Unlimit & ory 46 \\
\hline $\begin{array}{l}\text { 19. Security Classif. (of this report) } \\
\text { Unclassified }\end{array}$ & $\begin{array}{l}\text { 20. Security Classif. lof this page) } \\
\text { Unclassified }\end{array}$ & $\begin{array}{c}\text { 21. No. of pages } \\
9\end{array}$ & $\begin{array}{r}\text { 22. Price } \\
\text { A02 }\end{array}$ \\
\hline
\end{tabular}


National Aeronautics and

Space Administration

Code NTT-4

Washington, D.C.

20546-0001

BULK RATE

POSTAGE \& FEES PAID NASA

Permit No. G-27

Offlcial Business

Penalty for Private Use. 5300 